\title{
Two-Stage Degradation Assessment and Prediction Method for Aircraft Engine Based on Data Fusion
}

\author{
Hongsheng Yan $\left(\mathbb{D}\right.$, , Hongfu Zuo $\mathbb{D}^{\text {, }}$, Jianzhong Sun, Di Zhou, and Han Wang \\ Key Laboratory of Health Monitoring and Intelligent Maintenance, Nanjing University of Aeronautics and Astronautics, Nanjing, \\ Jiangsu Province 211106, China \\ Correspondence should be addressed to Hongfu Zuo; rms@nuaa.edu.cn
}

Received 14 June 2021; Revised 8 September 2021; Accepted 9 September 2021; Published 5 October 2021

Academic Editor: Joseph Majdalani

Copyright (c) 2021 Hongsheng Yan et al. This is an open access article distributed under the Creative Commons Attribution License, which permits unrestricted use, distribution, and reproduction in any medium, provided the original work is properly cited.

\begin{abstract}
Aeroengine is one of the most concerned objects of the relevant aviation industry and researchers, and it is a hard work to assess and predict performance degradation due to the complex structure and the changeable operating condition of the engine. In order to realize the performance degradation assessment and remaining useful life (RUL) prediction of aeroengine, this paper proposes a two-stage assessment and prediction method based on data fusion. First, the standard deviation merged by multiple selected features is used as the health indicator to characterize the engine performance. Second, a sliding window detection method called average local window slope is proposed to determine the current health state of observations by a specified rule. Finally, the RUL prediction is performed on the observation in the two stages, respectively. On the one hand, a similarity-based RUL prediction method is used to engines in the health stage, and on the other hand, for engines in the degradation stage, a RUL prediction method based on a mapping function of the standard deviation and the current using cycle is established. The proposed method has been applied and verified on the NASA's C-MAPSS simulation data. Results of degradation assessment and prediction show that the proposed method is trustworthy and feasible from the engineering perspective, and it has better performance in the comprehensive indicator compared with other methods.
\end{abstract}

\section{Introduction}

The aeroengine is the core component of modern military and civil aircraft, which performance will inevitably degrade with use. One thing is particularly concerned, performance degradation significantly shortens the operating reliability of engines, and leads to more serious safety risks and higher life-cycle costs (LCC) [1]. Effective performance degradation assessment and remaining useful life (RUL) prediction can help engineers make better maintenance decisions and support the realization of condition-based maintenance (CBM), which can help reducing operating cost and increasing the management and utilization of the fleet [2].

Due to the complex system composition and operation environment, performance degradation assessment and prediction technology of the engine have always been a hot research topic and a difficult problem in the research field related to the aviation industry. A review article comprehen- sively introduced technologies and methods of performance monitoring, diagnosis, and prediction of the engine in recent years, in which some critical research progress and major breakthroughs were summarized [3]. Studying a large number of related literature, the research of engine performance degradation assessment and prediction can be divided into three methods: model-based methods, data-driven methods, and deep learning (DL) methods. Generally, there are three critical technologies for performance degradation assessment and prediction of the engine: determining a characteristic indicator of performance degradation, establishing a trajectory function or model of degradation,; and proposing an effective RUL prediction approach.

For performance characterization, some researchers pay more attention to monitoring parameters of the engine itself and find important ones that can characterize the performance state, such as engine exhaust temperature margin (EGTM) [4, 5]. Another different approach is that a 
customized health indicator is used to characterize engine performance in some literature $[6,7]$. Comparing these two methods, the former one is intuitive and simple, but has certain limitation in applicability, because EGTM can only reflect the performance of the engine's gas path and is not suitable for characterizing the performance of the oil path and rotors. The latter approach is generally proposed for the quantitative degradation problem with a dimensionless health index quantity by reconstructing monitoring parameters; so, it is more adaptable and universal. One thing is important that the rationality of the customized performance indicator determines the accuracy of performance assessment and prediction. Some classic machine learning methods construct a health assessment indicator based on data fusion, which has been proven feasibility and convenience.

Degradation trajectory function or description model is the key to research on engine performance assessment and prediction and which is also an interdisciplinary research field involving physics, materials, and mathematics. Some classic distributions such as Gaussian process [8], wiener process [9], gamma process [10], and inverse Gaussian process [11] are used to describe engine performance degradation process. These methods are developed and verified on experimental data and simulation data, but there is an invite defect that these methods cannot meet actual engineering requirements. With the development of monitoring technology and data analysis method, research on engine performance degradation and RUL prediction is not only depends on restricted traditional degradation distributions but also using more and more abundant available monitoring data to realize data-driven model development. This type of data-driven engine physical degradation model takes into account both the description of the performance degradation process and the application of monitoring parameters. The most representative one is the two-stage degradation model [12]. As mechanical equipment and system, the use process of a general product includes the normal working phase and the failure delay phase; that is, there is a potential failure time point that changes the performance state from the normal working state to the defective state. Undoubtedly, RUL prediction should be different at different performance phases, but most of previous methods did not consider this.

Some researchers used data-driven methods to study engine life prediction problems. One thing is helpful: the complex physical degradation process does not need to be studied in depth. From previous studies, methods like random forest (RF), support vector machine (SVM), support vector regression (SVR), kernel principal component analysis (KPCA), and logistic regression (LR) are widely used [13-20]. However, this type of method has an inevitable flaw; that is, it requires a large amount of historical data and is not effective in early fault detection.

In addition to model-based and data-driven methods, deep learning-related methods are also used in the research of engine performance degradation assessment and prediction $[21,22]$. Some studies reported the use of the convolutional neural network (CNN) [23], long and short term memory network (LSTM) [24-26], and small-batch gradient descent neural network (GDN) [27] to the engine performance degradation evaluation and prediction. Unlike traditional methods that are limited by computing power and data availability, deep learning can implement supervised, unsupervised, and semisupervised training methods and output appropriate results. However, the interpretability of deep learning methods is poor, and the reliability and acceptability of the results always are the troublesome problem for dealing with engineering problems, especially engine performance evaluation and prediction. Generally, the interpretability of a model means engineering usability; that is, the model with better interpretability has a stronger generalization ability for practical problems [28]. Compared with the machine learning method, the interpretability of deep learning method is not obvious.

Although there are many existing methods for engine performance assessment and prediction, there are still no methods that take into account early fault detection, model interpretability, and multistage life prediction. In order to fill gaps in related research fields and solve practical engineering application problems, in this paper, a two-stage aeroengine degradation assessment and prediction method based on data fusion is proposed, and the innovation of this study is highlighted as follows: (1) a novel comprehensive health index (standardized deviation, SD) based on the consistent trend of mutiparameters is used; (2) a two-stage degradation assessment method is proposed and realized the time window to find the first occurrence of potential degradation, which is surprisingly able to find early failures in the process of engine performance degradation; (3) for observations in the health stage, a RUL prediction method based on the similarity of historical samples is proposed; (4) for observations in the degradation stage, a mapping function based on the health index and usage time is established, and RUL prediction is realized based on it.

The rest of this article is arranged as follows. In Section II, the theoretical background related to degradation assessment and prediction is introduced, and general definitions and introductions of the proposed method are expressed. In Section III, the proposed method is highlighted in detail, including the calculation process and evaluation function of degradation assessment and RUL prediction. In Section IV, an application case based on NASA's turbine engine simulation data (CMAPSS) is presented, and the results are compared with other methods in related literature. Finally, the conclusion is given in Section V.

\section{Theoretical Background}

2.1. Degradation Process of the Aircraft Engine. The performance degradation of mechanical equipment can be divided into two types: wear type of lifetime and non-wear of lifetime. The performance of the former type will decrease irreversibly because of using without effective maintenance. The performance degradation of the latter type has nothing to do with the use time, and which shows generally as a random failure.

For the mechanical equipment that meets the first type, the classic P-F curve describes the degradation process. 
Generally speaking, since installment, there will be some potential failures occur after a period of running-in time of the mechanical equipment, but it will not cause function failure or unexpected shutdown. However, if the equipment keeps working in a defective state without any treatment when a potential failure occurs, it will accelerate the speed of performance degradation and cause a functional failure and shutdown maintenance. The P-F curve of mechanical equipment is shown in Figure 1.

Aeroengine is a complex system composed of multiple mechanical components, but it is also a typical wearable mechanical equipment; that is, the degradation process of the engine conforms to the $P-F$ curve. The main structure diagram of the engine [29] is shown in Figure 2.

In order to realize the condition-based maintenance of the engine, it is very important to find the $P$-point where the performance is significantly degraded or abnormal. The period before $P$-point can be understood as a healthy working phase, and there is a defective working time with a potential failure after P-point. Therefore, the two-stage degradation model is pretty meaningful for describing engine performance degradation.

2.2. Definition of the State of Health. Lifetime data is important source for reliability evaluation and degradation prediction; besides, it is easily collected by operators. For components assembled on the aircraft, time since installment (TSI) is the typical common life data, which represents the real service time of a component or a system since installment operation. Moreover, TSI can be as the time scale to evaluate the health state of the observation object.

There is no doubt that there is a negative correlation between TSI and RUL; that is, the sum of the two is equal to the total life. In order to quantitatively characterize this negative correlation, this paper proposes a dimensionless index, named state of health ( $\mathrm{SOH})$. The advantage of $\mathrm{SOH}$ is to establish a remaining life prediction method based on the mapping function. The SOH can be calculated by (1) at time $t$.

$$
\mathrm{SOH}_{t}=\left(L-\mathrm{TSL}_{t}\right) / L
$$

where $\mathrm{TSI}_{t}$ is the service time since installment at the time $t$, and $L$ is the actual life of the observation object. So, the $\mathrm{SOH}$ is the ratio between RUL and the all lifetime, and the value range of SOH is $[0,1)$. Obviously, the RUL is the difference between the all lifetime and the nominal use time of the current time. One thing is important, at any time, the all lifetime is unknown (except for the final removal time), but the TSI is obtainable, and the $\mathrm{SOH}$ is associated with monitoring parameters that characterize performance status. Therefore, for a component or a system with the significant degradation process, the RUL at any time $t$ can be calculated by (2).

$$
\mathrm{RUL}_{t}=\mathrm{TSI}_{t}^{*} \frac{\mathrm{SOH}_{t}}{1-\mathrm{SOH}_{t}}
$$

2.3. Summary of Previous Works Related to CMAPSS. In addition to the performance degradation process and the $\mathrm{SOH}$ definition of the engine, the stable and representative data is the

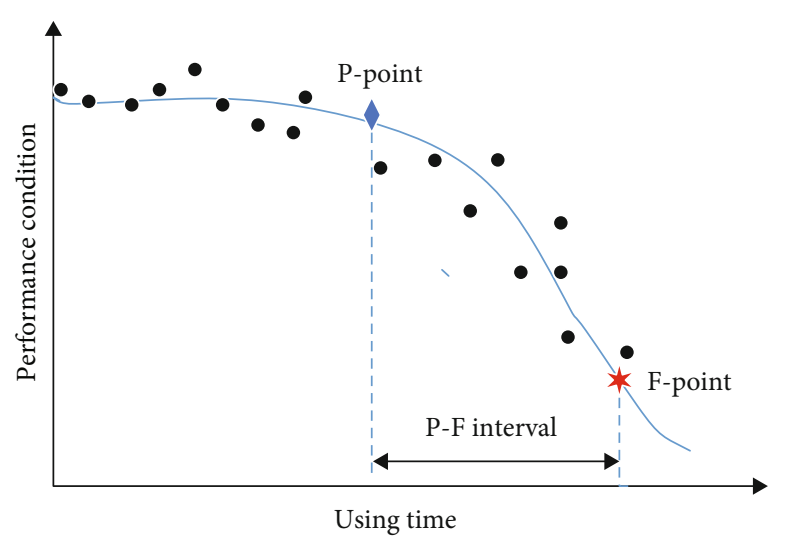

- Raw data

Figure 1: $P-F$ curve of general wearable mechanical equipment.

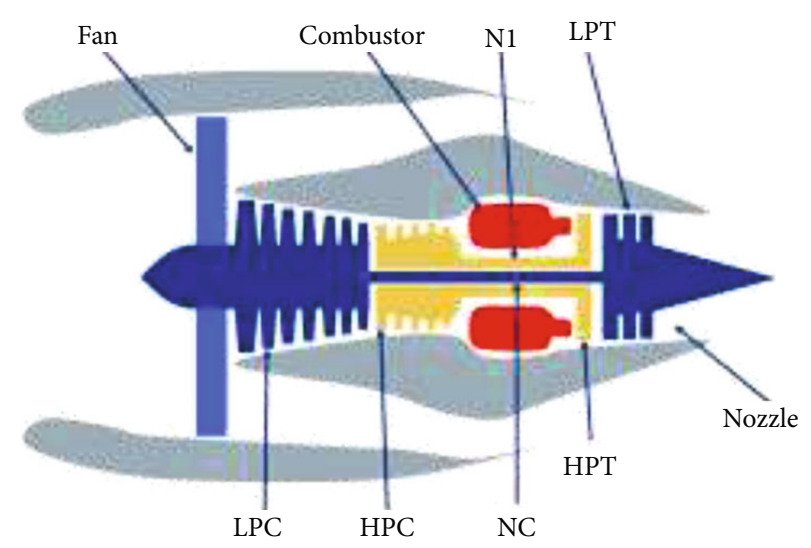

FIgURE 2: The structure diagram of the aircraft engine.

most important condition for modeling and verification. Generally, the available and complete real-world data is best for modeling and verification. However, for performance assessment and RUL prediction of the aeroengine, there are two main difficulties in obtaining real-world data: (1) obtaining real-world data is expensive; (2) it is very difficult to guarantee the integrity and quality of all-life-cycle real-world data. NASA developed a tool for the simulation of a realistic large commercial turbofan engine, named "Commercial Modular AeroPropulsion System Simulation" (C-MAPSS). Although the CMPASS data is generated by simulation, the simulation is designed based on the physical mechanism and degradation process of a realistic large commercial turbofan engine. Many studies were reported to performance assessment and RUL prediction for the engine based on C-MAPSS. By summarizing the related literature, these methods in recent years can be classified into three types: statistical-based methods, machine learning, and deep learning, as shown in Table 1.

Our proposed method is used to evaluate the health stage and RUL prediction by deviations of the performance parameters, which is driven by data rather than relying on physical models or principles; so, the CMAPSS data can meet the needs of model verification. The details of the proposed method are presented in Section III. 
TABLE 1: Summary of previous works related to CMAPSS.

\begin{tabular}{lccc}
\hline Type & Method & Purpose & Reference \\
\hline & KNN + Bayesian filter & RUL prediction & Mosallam et al. [30] \\
Statistical-based & Entropy-based & Condition monitoring and & Lrognostics \\
& Signal fusion + stochastic degradation model & RUL prediction & Wen et al. [32] \\
& PSO + SVM & RUL prediction & García Nieto et al. [15] \\
Machine & ARIMA+SVM & RUL prediction & Ordóñez et al. [16] \\
learning & LR + OS-ELM & Degradation prognostics & Lu et al. [18] \\
& Deep RNN & Degradation prognostics & Behera, Misra, and Sillitti [33] \\
LSTM+GMM & RUL prediction & Sayah et al. [34] \\
Deep learning & Bidirectional handshaking LSTM & RUL prediction & Elsheikh, Yacout, and Ouali [35] \\
& MLE + CCF & Predictive maintenance & Pillai and Vadakkepat [36] \\
\hline
\end{tabular}

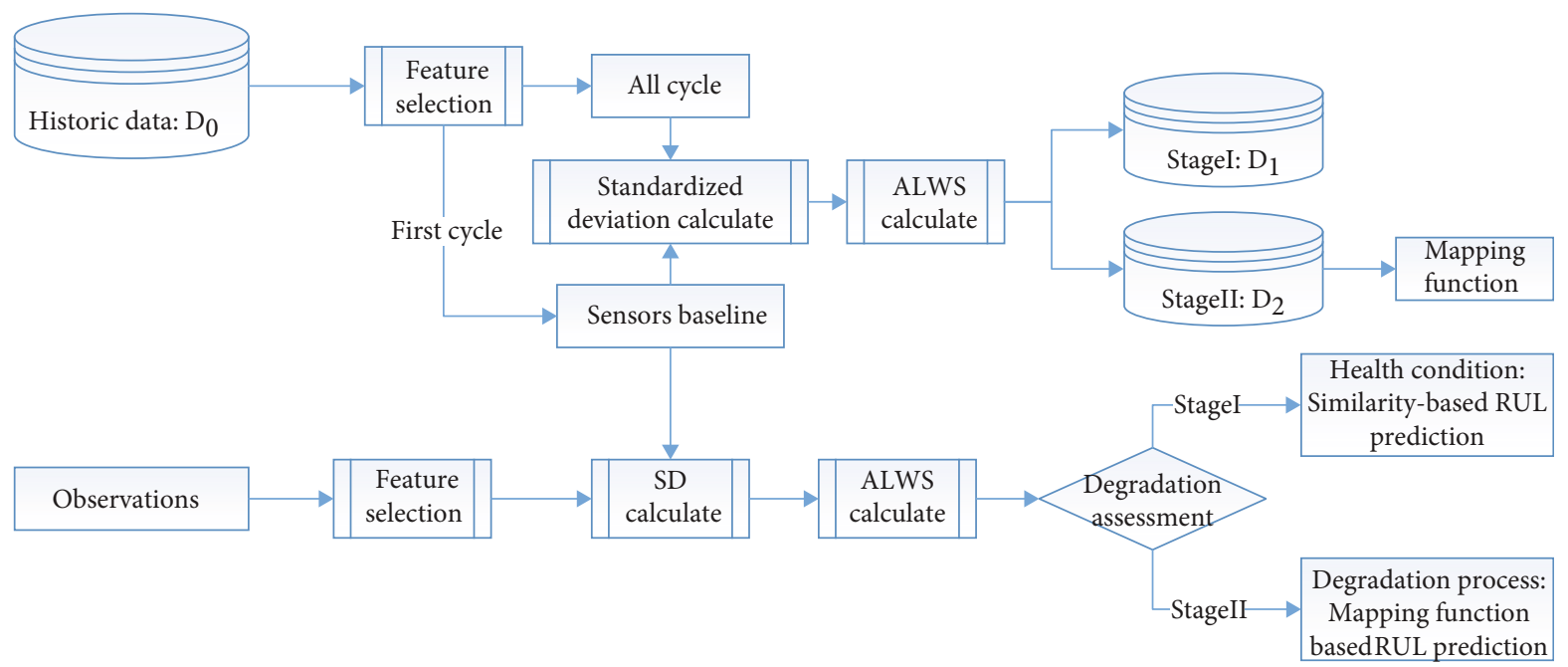

FIGURE 3: Overall framework for degradation assessment and RUL prediction.

\section{Proposed Methodology and Algorithms}

3.1. General Introduction of the Proposed Method. The proposed two-stage aeroengine degradation assessment and prediction method based on data fusion is shown in Figure 3.

Totally, the proposed method includes historical data processing and analysis, defined indicators calculation, degradation assessment and RUL prediction, and specific definitions and calculations are emphasized in section B.

3.2. Definition and Calculation. In order to get a better performance of assessment and RUL prediction, the fundamental and important work is to select appropriate features. In this paper, based on the results of trend analysis, in all cycle data of a single engine, the parameter that has the obvious monotonic change trend with time is selected. There are two advantages: one is to remove parameters that are not related to performance degradation; the other is to avoid the influence of parameters with inconsistent trends on evaluation and prediction. Mann-Kendall is a common monotonousness detection method, which can realize rapidly

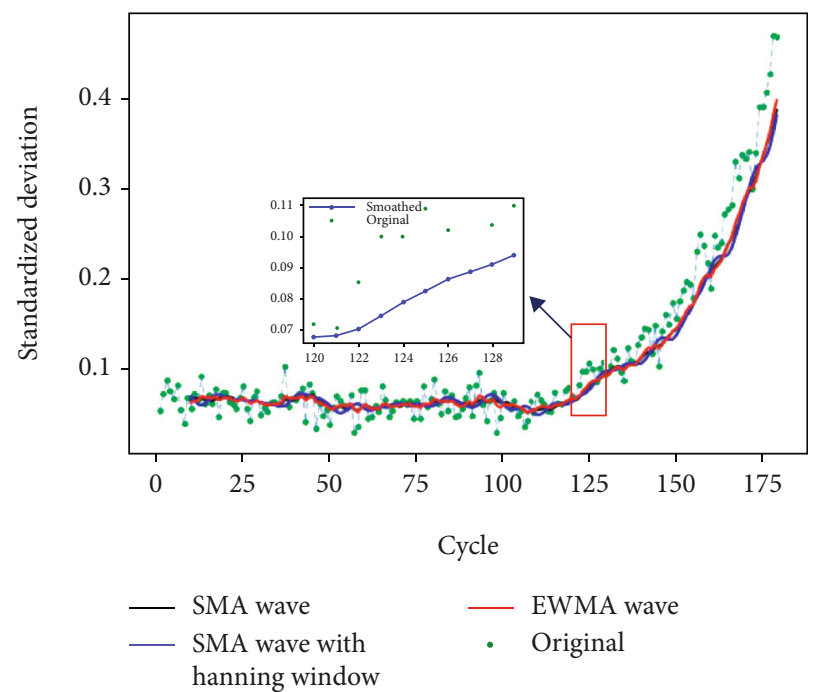

Figure 4: SD sequence of a sample with full cycles. 
The degradation detection for historical datasets.

Step1. Initialization. L represents the moving detection window length, $w$ represents the window width of ALWS, and let $\mathrm{L}=\mathrm{L} 1, w=w 1$.

Step2. According formula(3), calculate the $\mathrm{SD}$ sequence $\left(\left[\mathrm{SD}_{1}, \mathrm{SD}_{2}, \ldots, \mathrm{SD}_{N}\right]\right)$ of full cycles.

Step3. Calculate the ALWS sequence of full cycles.

For $i$ in $(w, N)$ :

$\mathbf{s d}=\left[\mathrm{SD}_{i-\mathrm{w}+1}, \mathrm{SD}_{i-\mathrm{w}+1}, \ldots, \mathrm{SD}_{i}\right]$

According the formula (), calculate $\mathrm{a}(\mathrm{i})=\mathrm{ALWS}(\mathbf{s d})$

End for

Get the sequence $\left[a_{1}, a_{2}, \ldots, a_{N-w+1}\right]$. Add m-1 zero before it and get the new ALWS sequence $\left[A_{1}, A_{2}, \ldots, A_{m-1}, a_{1}, \ldots a_{N-w+1}\right]$, where $\mathrm{A}_{1}=\ldots=\mathrm{A}_{\mathrm{w}-1}=0$.

Step4. Using $Z$ to represent the number of nonzeros in the detection window sequence and [T_low, $T$ _up] represents the time interval when the obvious degradation point.

For $\mathrm{j}$ in $(1, \mathrm{~N}-\mathrm{L})$, do:

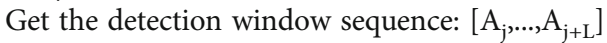

Count the number $Z$ of nonzero in the detection sequence: $Z=$ is_not_zero $\left(\left[A_{j}, \ldots, A_{j+L}\right]\right)$

If $\mathrm{Z}$ more than int $\left(0.5^{*} \mathrm{~L}\right)$

T_low $=\mathrm{j}$

T_up $=\mathrm{j}+\mathrm{L} 1$

break

Else

continue

End if

End for

Step5. For each engine in the historic database, according to the above step 1-4, the degradation interval is determined, and the original historical database D0 can be divided into two datasets, D1 in the health stage and D2 in the obvious degradation stage.

Pseudocode 1: The pseudocode of degradation detection for historical datasets.

\section{The degradation detection for new observations}

Step1. Initialization, if $\mathrm{K}>100, \mathrm{~L}=\mathrm{L} 1$ and $\mathrm{w}=\mathrm{w} 1$; else $\mathrm{L}=\mathrm{w}=\mathrm{w} 2$. And score sequence $\mathbf{S}=[\mathrm{]}$

Step2. Based on the health stage datasets D1, calculating the ALWS(D1) sequence, and taking the maximum value max(ALWS(D1)) as the threshold e_upper.

Step3. Calculating ALWS $(\mathbf{X})$ sequence $\mathbf{A}$ and adding w-1 zeros before it. Getting the new sequence $\mathbf{A}:\left[\mathrm{A}_{1}, \mathrm{~A}_{2}, \mathrm{~A}_{\mathrm{w}-1}, \mathrm{~A}_{\mathrm{w}}, \ldots, \mathrm{A}_{\mathrm{K}}\right]$ with length $\mathrm{K}$, and $\mathrm{A}_{1}=\mathrm{A}_{2}=\ldots=\mathrm{A}_{\mathrm{w}-1}=0$.

Step4. Updating sequence $\mathbf{A}\left(\left[\mathrm{A}_{1}, \mathrm{~A}_{2}, \mathrm{~A}_{\mathrm{w}-1}, \mathrm{~A}_{\mathrm{w}}, \ldots, \mathrm{A}_{\mathrm{K}}\right]\right)$.

For a in A, do:

If a less than or equal to e_upper:

$\mathrm{a}=0$.

Else:

Continue

End if

End for

Step5. Degradation assessment for the observation.

For $\mathrm{i}=\mathrm{L}, 2, . ., \mathrm{K}$, do:

Get detecting window $\left[\mathrm{A}_{\mathrm{i}-\mathrm{L}+1}, \ldots, \mathrm{A}_{\mathrm{i}}\right]$

Count the number $S_{i}$ of zeros in $\left[A_{i-L+1}, \ldots, A_{i}\right]$

Add new element into the score sequence $\mathbf{S}, \mathbf{S}=\mathbf{S}+\mathrm{S}_{\mathrm{i}}$

End for

If $\max (\mathbf{S})$ more than int $(0.5 * \mathrm{~L})$ :

This observation is in the degradation stage.

Else:

This observation is in the health stage.

Pseudocode 2: The pseudocode of degradation detection for new observations. 
TABLE 2: Description of CMAPSS simulation data.

\begin{tabular}{lcc}
\hline Data set & Operation environment & Fault mode \\
\hline FD001 & 1 & 1 \\
FD002 & 6 & 1 \\
FD003 & 1 & 2 \\
FD004 & 6 & 2 \\
\hline
\end{tabular}

TABle 3: Parameter types and description in datasets.

\begin{tabular}{lccc}
\hline Symbol & & Description & Units \\
\hline Id & & Engine number & - \\
Cycle & & Current engine cycle & - \\
Setting1 & & Operation parameter setting 1 & - \\
Setting2 & & Operation parameter setting 2 & - \\
Setting3 & & Operation parameter setting 3 & - \\
Sensor parameters available for analysis & \\
S1 & T2 & Total temperature at fan inlet & ${ }^{\circ} \mathrm{R}$ \\
S2 & T24 & Total temperature at LPC outlet & ${ }^{\circ} \mathrm{R}$ \\
S3 & T30 & Total temperature at HPC outlet & ${ }^{\circ} \mathrm{R}$ \\
S4 & T50 & Total temperature at LPT outlet & ${ }^{\circ} \mathrm{R}$ \\
S5 & P2 & Pressure at fan inlet & Psia \\
S6 & P15 & Total pressure in bypass-duct & Psia \\
S7 & P30 & Total pressure at HPC outlet & Psia \\
S8 & Nf & Physical fan speed rpm & Rpm \\
S9 & Nc & Physical core speed rpm & Rpm \\
S10 & Epr & Engine pressure ratio (P50/P2) & - \\
S11 & Ps30 & Static pressure at HPC outlet & Psia \\
S12 & Phi & Ratio of fuel flow to Ps30 & Pps/psi \\
S13 & NRf & Corrected fan speed & Rpm \\
S14 & NRc & Corrected core speed & Rpm \\
S15 & BPR & Bypass ratio & - \\
S16 & farB & Burner fuel-air ratio & - \\
S17 & htBleed & Bleed enthalpy & - \\
S18 & Nf_dmd & Demanded fan speed & Rpm \\
S19 & PCNfR_dmd & Demanded corrected fan speed & Rpm \\
S20 & W31 & HPT coolant bleed & Lbm/s \\
S21 & W32 & LPT coolant bleed & Lbm/s \\
\hline & & &
\end{tabular}

simultaneous detection for multiple parameters. This paper used the Mann-Kendall method select features and proposed the required characteristics from the original parameter set based on the detection results.

With selected features, the baseline can be determined by the specific cycle. In this paper, features at the first cycle of all engines constitute the considered absolute health set, denoted as $\mathbf{H}$. Obviously, the mean value of $\mathbf{H}$ can be used as the healthy baseline.

\subsection{Definition and Calculation of Standardized Deviation} $(S D)$. In existing studies, there are three methods for constructing health indicator (HI) for state evaluation: one is to directly select sensor parameter as HI, the other is constructing $\mathrm{HI}$ based on deviation between the original input
TABLE 4: Results of Mann Kendall test for all parameters in FD001.

\begin{tabular}{lcccc}
\hline Sensor & Trend & Hypothesis & $P$ value & Score \\
\hline S1 & No trend & False & 1.0 & 0.0 \\
S2 & Increasing & True & $\leq \mathbf{0 . 0 0 1}$ & $\mathbf{9 0 6 6 . 0}$ \\
S3 & Increasing & True & $\mathbf{4 . 4 4 e - 1 6}$ & $\mathbf{7 2 1 2 . 0}$ \\
S4 & Increasing & True & $\leq \mathbf{0 . 0 0 1}$ & $\mathbf{1 1 7 9 6 . 0}$ \\
S5 & No trend & False & 1.0 & 0.0 \\
S6 & No trend & False & 1.0 & 0.0 \\
S7 & Decreasing & True & $\leq \mathbf{0 . 0 0 1}$ & $\mathbf{- 1 0 8 6 5 . 0}$ \\
S8 & Increasing & True & $\leq \mathbf{0 . 0 0 1}$ & $\mathbf{1 1 2 1 1 . 0}$ \\
S9 & Decreasing & True & $\mathbf{2 . 5 8 e - 0 9}$ & $\mathbf{- 5 3 0 3 . 0}$ \\
S10 & No trend & False & 1.0 & 0.0 \\
S11 & Increasing & True & $\leq \mathbf{0 . 0 0 1}$ & $\mathbf{1 2 1 7 2 . 0}$ \\
S12 & Decreasing & True & $\leq \mathbf{0 . 0 0 1}$ & $\mathbf{- 1 1 9 1 0 . 0}$ \\
S13 & Increasing & True & $\leq \mathbf{0 . 0 0 1}$ & $\mathbf{1 1 8 5 9 . 0}$ \\
S14 & Decreasing & True & $\leq \mathbf{0 . 0 0 1}$ & $\mathbf{- 9 2 6 9 . 0}$ \\
S15 & Increasing & True & $\leq \mathbf{0 . 0 0 1}$ & $\mathbf{9 4 0 9 . 0}$ \\
S16 & No trend & False & 1.0 & 0.0 \\
S17 & Increasing & True & $\leq \mathbf{0 . 0 0 1}$ & $\mathbf{8 6 5 3 . 0}$ \\
S18 & No trend & False & 1.0 & 0.0 \\
S19 & No trend & False & 1.0 & 0.0 \\
S20 & Decreasing & True & $\leq \mathbf{0 . 0 0 1}$ & $\mathbf{- 9 6 5 1 . 0}$ \\
S21 & Decreasing & True & $\leq \mathbf{0 . 0 0 1}$ & $\mathbf{- 1 0 4 8 7 . 0}$ \\
\hline & & & &
\end{tabular}

and the health baseline, and the third is constructing $\mathrm{HI}$ based on data fusion model such as the autoencoding model.

Comprehensively, the first method is difficult to find the sensor parameter that directly characterizes the degradation, and the third HI construction method has the disadvantage of complex calculation and low applicability. In contrast, the second deviation-based HI construction method is better.

Deviation is direct and accessible to show the difference between the new observation and the healthy baseline. However, one latent mistake will affect the correctness of the healthy baseline that some extreme values of wrong recording or invalid will be used in $H$, because the healthy baseline is the mean value of health set $H$, and $H$ comes from historic database D0. In this paper, we use the amplitude value of the individual parameter in $H$ to correct deviation, and for multivariate parameters, define a dimensionless value standardized deviation to represent the difference between the observation and the baseline, denoted as SD.

$H$ with $n$ features is represented as $H=\left\{S_{1}, S_{2}, \cdots, S_{n}\right\}$; thus, the healthy baseline is mean $(H)$, and the parameter amplitude value in $H$ is $(\max (H)-\min (H))$. For the observation data with $n$ parameters, expressed as $X=\left[x_{1}, x_{2}, \cdots, x_{n}\right]$, the standard deviation $\mathrm{SD}$ can be calculated by the following (3).

$$
\mathrm{SD}=\frac{1}{n} \times \sum_{i=1}^{n}\left(\operatorname{Sign}(i) \cdot\left(\frac{X-\operatorname{mean}(H)}{\max (H)-\min (H)}\right)\right),
$$

where $\operatorname{sign}(i)$ represents identification function of the monotonic trend of the $i$ th feature. If it is increased, sign $=1$; on the contrary, sign $=-1$. 

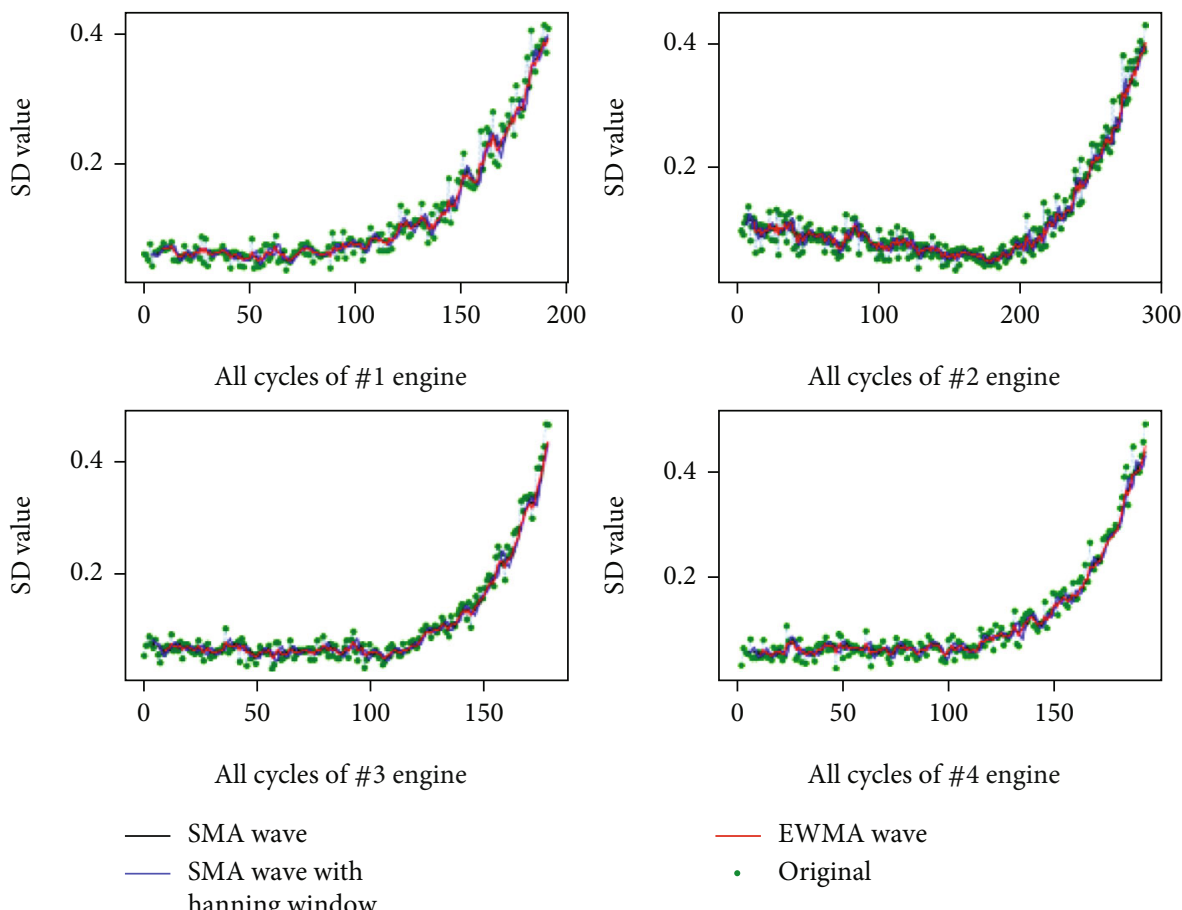

Figure 5: SD value sequence of full-cycle samples in D0.

Considering some random errors in monitoring data, the SD sequence should be smoothed before analyzing. There three commonly used smoothing methods: simple moving average (SMA), SMA with Hanning window, and exponentially weighted moving average (EWMA). After processing a sample as shown in Figure 4, it is found that three smoothed results are basically same, but SMA is easy and efficient in processing speed; so, SMA is used as the smoothing method in this paper.

3.3.1. Definition and Calculation of Average Local Window Slope (ALWS). SD is an effective indicator for state evaluation, but it is not sensitive to early degradation warning, which means that it is difficult to find the two-stage change interval. In order to find the time point of the state change in the degradation process, this paper uses average local window slope (ALWS) as the detection index. ALWS is the corrected slope of the SD sequence in a certain time length. According to Figure 4, the trend of SD shows as consistently increasing. For any time $t$, the time window set is $\left\{\mathrm{SD}_{t-w}, \mathrm{SD}_{t-w+1}, \cdots\right.$, $\left.\mathrm{SD}_{t}\right\}$, the mean value of first $w$-1 data is used as the first element, the $w$-th data $\mathrm{SD}_{\mathrm{t}}$ is used as the second element, and ALWS at time $t$ is calculated by the following (4).

$$
\operatorname{ALWS}(t)=\left\{\begin{array}{l}
0, \text { if } S D_{t}-\operatorname{mean}\left(\left[\mathrm{SD}_{t-w}, \mathrm{SD}_{t-w+1}, \cdots, \mathrm{SD}_{t-1}\right]\right)<0 \\
\frac{S D_{t}-\operatorname{mean}\left(\left[\mathrm{SD}_{t-w}, \mathrm{SD}_{t-w+1}, \cdots, \mathrm{SD}_{t-1}\right]\right)}{w}, \text { else if }
\end{array} .\right.
$$

\subsection{Degradation Assessment}

3.4.1. For Historic Database. For any engine with full use cycles and the number of cycles of this engine is $N$, the pro- cess of degradation detection called the SD-ALWS method and is as the Pseudocode 1.

3.4.2. For New Observations. For any observation $X$ with $K$ elements, the process of degradation detection is shown in Pseudocode 2 .

3.5. Remaining Useful Life Prediction. According to the result of degradation assessment, a similarity-based method is used to predict the remaining life of the observation which in the healthy stage. And for the observation in the obvious degradation stage, the mapping function based on $\mathrm{SOH}$ and $\mathrm{SD}$ is used to calculate the remaining life.

3.5.1. Similarity-Based RUL Prediction for Stage 1 (Observations in Health Condition). For observations in health condition, we proposed the similarity-based RUL prediction method. The similarity-based method is a general data driven approach [37]. $X$ represents the observation, \{ $\left.Y_{1}, Y_{2}, \cdots, Y_{M}\right\}$ represents the historical samples, $\left\{T_{1}, T_{2}\right.$, $\left.\cdots, T_{M}\right\}$ represents the true used cycles of historic engines, and $M$ is the engine number in D0. The RUL of $X$ can be calculated by the following equation (5).

$$
\operatorname{RUL}(X)=\sum_{i=1}^{M} w\left(X, Y_{i}\right) * T_{i}=\sum_{i=1}^{M} C(i) * R\left(X, Y_{i}\right) * T_{i}
$$

where $w\left(X, Y_{i}\right)$ represents the weight of the $i$ th historical sample that predicts the RUL of the observation in the healthy state, $C(i)$ represents the survival rate corresponding to the life value of the $i$ th historical sample, and $R\left(X, Y_{i}\right)$ represents the similarity between the observation and the $i$ th sample. 

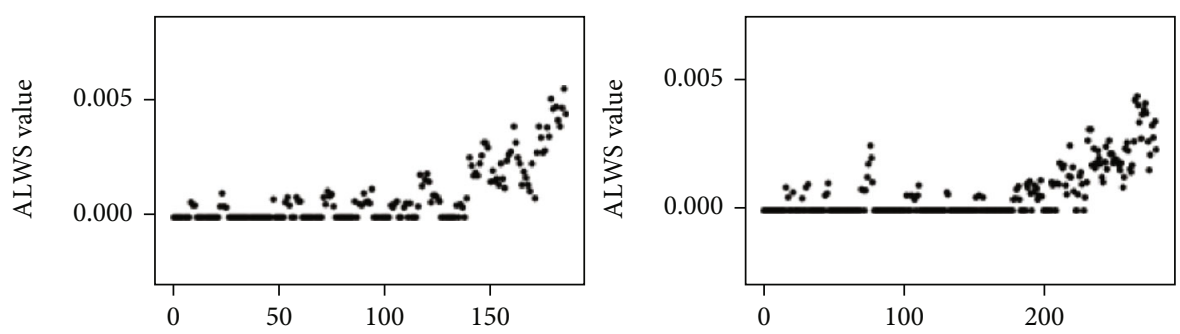

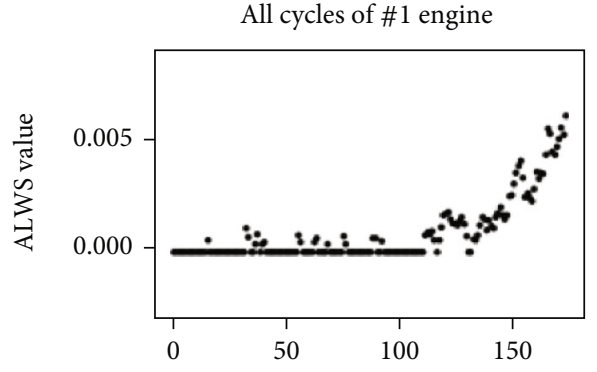

All cycles of \#3 engine

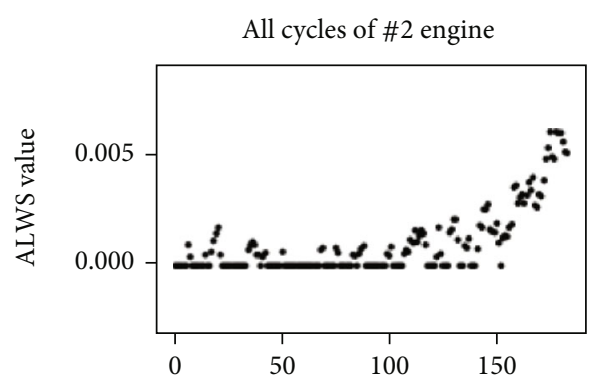

All cycles of \#4 engine

Figure 6: ALWS value of full-cycle samples in D0.

In order to ensure the reasonableness of RUL prediction, the survival analysis based on the Kaplan Meier model is performed on the historical samples, and the survival rate corresponding to each life value is the reliability $C(i)$.

There are many studies on similarity, including point to point, point to sample, and sample to sample. Generally, distance and KL divergence are used as the measurement index. However, for high-dimensional samples, distance or KL divergence cannot perform well in the two-sample test. The literature introduces common methods for the twosample test. Satisfactorily, the maximum mean discrepancy (MMD) is an excellent method that can solve the problem of high-dimensional samples [38]. In this paper, MMD is selected as the index of the similarity between the observation and historical samples, $H\left(Y_{\mathrm{i}}\right)(i=1, \cdots, M)$ is used to represent the length of used cycles in the health stage of the historical sample $\boldsymbol{Y}_{i}$, and $\boldsymbol{Y}_{i}[0: \operatorname{TSI}(\boldsymbol{X})]$ is used to represent the historical sample $\boldsymbol{Y}_{i}$ compressed to the same length of cycles as the observation. The similarity weight is calculated by the following equation (6).

$$
R\left(X, Y_{i}\right)=\left\{\begin{array}{ll}
0, & \mathrm{H}\left(Y_{i}\right)<\operatorname{TSI}(X) \\
\operatorname{MMD}\left(X, Y_{i}[0: \operatorname{TSI}(X)]\right), & \mathrm{H}\left(Y_{i}\right) \geq \operatorname{TSI}(X)
\end{array}, i=1, \cdots, M .\right.
$$

For two samples $p$ and $q$, the definition of MMD [39] is shown in (7).

$\operatorname{MMD}[\mathscr{F}, p, q]:=\sup _{f \in \mathscr{F}}\left(E_{x \sim p}[f(x)]-E_{y \sim q}[f(y)]\right)=\sup _{f \in \mathscr{F}}\langle f, \mu p-\mu q\rangle_{\mathscr{H}}$

where $\mathscr{F}$ represents the unit ball in reproducing Hilbert kernel space $($ RHKS, $\mathscr{H}), f(\cdot)$ represents the data mapping function to RHKS, and $\mu$ represents the expected value of sample.

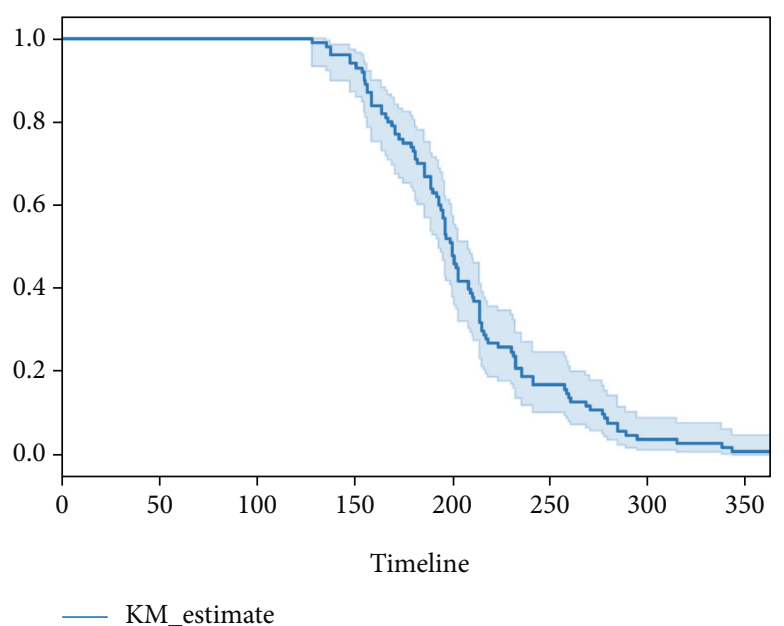

FIgURE 7: The diagram of survival analysis for the historical life data.

Let $Z=Y[0: \operatorname{TSI}(X)]$, and (7) can be solved by (8).

$$
\begin{aligned}
\operatorname{MMD}^{2}(\mathbf{X}, \mathbf{Z})= & \left\|\frac{1}{n} \sum_{i=1}^{n} f\left(x_{i}\right)-\frac{1}{m} \sum_{j=1}^{m} f\left(z_{j}\right)\right\|_{\mathscr{H}}^{2} \\
= & \| \frac{1}{n^{2}} \sum_{i=1}^{n} \sum_{j \neq i}^{n} f\left(x_{i}\right) f\left(x_{i}\right)-\frac{2}{n m} \sum_{i=1}^{n} \sum_{j=1}^{m} f\left(x_{i}\right) f\left(z_{j}\right) \\
& +\frac{1}{m^{2}} \sum_{i=1}^{m} \sum_{j \neq i}^{m} f\left(z_{j}\right) f\left(z_{j}\right) \|_{\mathscr{H}}^{2}
\end{aligned}
$$

With kernel trick, we skip calculating function $\mathrm{f}$ and using $k\left(x_{i}\right) k\left(x_{j}\right)$ instead, and $k(\cdot)$ represents the kernel function. 
TABLE 5: Function forms and parameters estimation of fitting results.

\begin{tabular}{|c|c|c|c|}
\hline Function form & Mathematical expression & Parameter estimation & $R$-square \\
\hline Quadratic polynomial & $y=p_{i} * x+p_{2} * x^{2}+p_{3}$ & $\begin{array}{c}p_{1}=-1.99 \\
p_{2}=1.95 \\
p_{3}=0.51\end{array}$ & 0.71 \\
\hline Inverse function & $y=p_{1}+p_{2} / x$ & $\begin{array}{c}p_{1}=-0.079 \\
p_{2}=0.049\end{array}$ & 0.7 \\
\hline Power function & $y=p_{1} * x^{p_{2}}+p_{3}$ & $\begin{array}{c}p_{1}=-12.45 \\
p_{2}=0.019 \\
p_{3}=12.25\end{array}$ & 0.73 \\
\hline Negative exponential & $y=\exp \left(-p_{1} * x\right)+p_{2}$ & $\begin{array}{c}p_{1}=1.33 \\
p_{2}=-0.58\end{array}$ & 0.69 \\
\hline Logarithmic function & $y=p_{1}+p_{2} * \operatorname{Ln}(x)$ & $\begin{array}{l}p_{1}=-0.195 \\
p_{2}=-0.234\end{array}$ & 0.74 \\
\hline Hybrid function & $y=\exp \left(-p_{1} * x\right)+p_{2} / x+p_{3} * \operatorname{Ln}(x)$ & $\begin{array}{c}p_{1}=4.82 \\
p_{2}=0.015 \\
p_{3}=0.145\end{array}$ & 0.76 \\
\hline
\end{tabular}

Unbiased value can be calculated by (9).

$$
\begin{aligned}
\operatorname{MMD}_{u}^{2}(X, Z)= & \| \frac{1}{n(n-1)} \sum_{i=1}^{n} \sum_{j \neq i}^{n} k\left(x_{i}, x_{j}\right)-\frac{2}{n m} \sum_{i=1}^{n} \sum_{j=1}^{m} k\left(x_{i}, z_{j}\right) \\
& +\frac{1}{m(m-1)} \sum_{i=1}^{m} \sum_{j \neq i}^{m} k\left(z_{i}, z_{j}\right) \|_{\mathscr{H}}^{2}
\end{aligned}
$$

3.5.2. Mapping Function-Based RUL Prediction for Stage II (Observations in Degradation Stage). For engines in the degradation process, different objects will have unique gradation trajectories; so, the similarity-based RUL prediction method is not suitable for this problem. In this paper, the mapping function-based RUL prediction method is proposed for observations in degradation process.

Generally, there are several difficulties in directly constructing the functional relationship between multiple performance parameters and $\mathrm{SOH}$ : one is that this relationship is difficult to express by a known functional form; another problem is that the accuracy of the functional relationship established directly using original performance parameters with random errors is poor. According to the above introduction and calculation process about the fusion parameter SD of multiple performance parameters, it can be seen that SD can completely characterize the performance degradation process, and in the obvious degradation stage, there is a mapping function that can be expressed by a mathematical model with $\mathrm{SOH}$.

The mapping function is an abstract expression about the relationship between $\mathrm{SD}$ and $\mathrm{SOH}$. Exactly, there is no fixed mathematical expression for the mapping function. According to the fitting result of datasets of SD and $\mathrm{SOH}$, optimal mapping function is determined, and function parameters are estimated by the Levenberg-Marquardt method.

$\theta(\cdot)$ is used to represent the mapping function between $\mathrm{SD}$ and $\mathrm{SOH}$, and for any using cycle time $t$, the observation

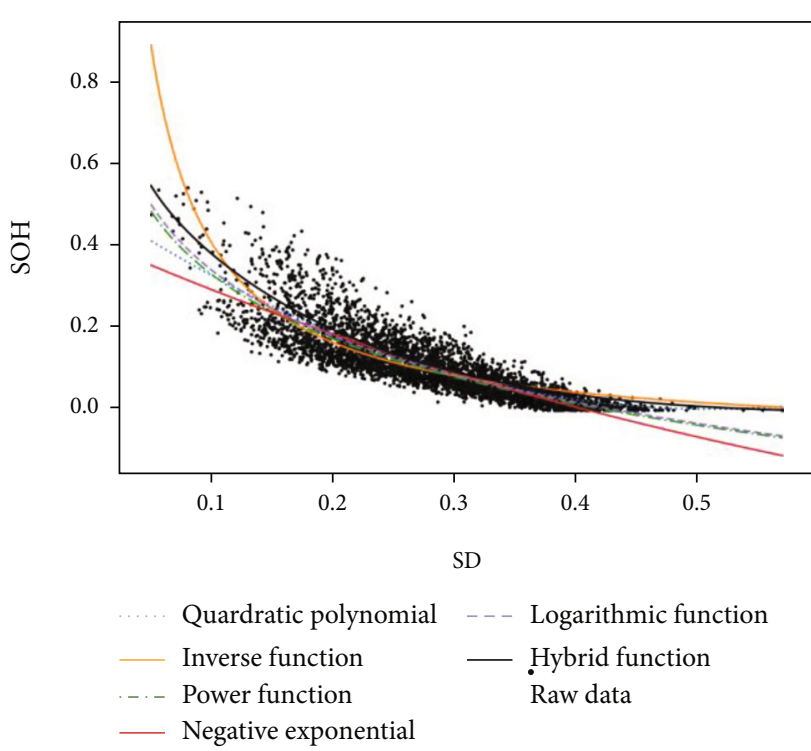

FIGURE 8: Drawing diagram of fitting results.

at time $\mathrm{t}$ is $\boldsymbol{X}_{t}, \mathrm{SOH}$ can be represented as $\mathrm{SOH}\left(X_{t}\right)=\theta($ $\left.\mathrm{SD}\left(X_{t}\right)\right)$.

With the formula, RUL at time $t$ of the new observation can be calculated by (10).

$$
\mathrm{RUL}=\mathrm{TSI}_{t}^{*} \frac{\mathrm{SOH}_{t}}{1-\mathrm{SOH}_{t}}=\mathrm{TSI}_{t}^{*} \frac{\theta\left(\mathrm{SD}_{t}\right)}{1-\theta\left(\mathrm{SD}_{t}\right)}
$$

where $\mathrm{TSI}_{t}$ represents time since installment at time $t$, $\mathrm{SOH}_{t}$ represents the $\mathrm{SOH}$ value at time $t, \theta(\mathrm{SD})$ represents the mapping function of $\mathrm{SD}$ and $\mathrm{SOH}$, and $\mathrm{SD}_{t}$ represents the SD value at time $t$.

3.6. Evaluation Metric. A reasonable evaluation metric is necessary to improve and optimize the proposed assessment and prediction method. Generally, for methods of 

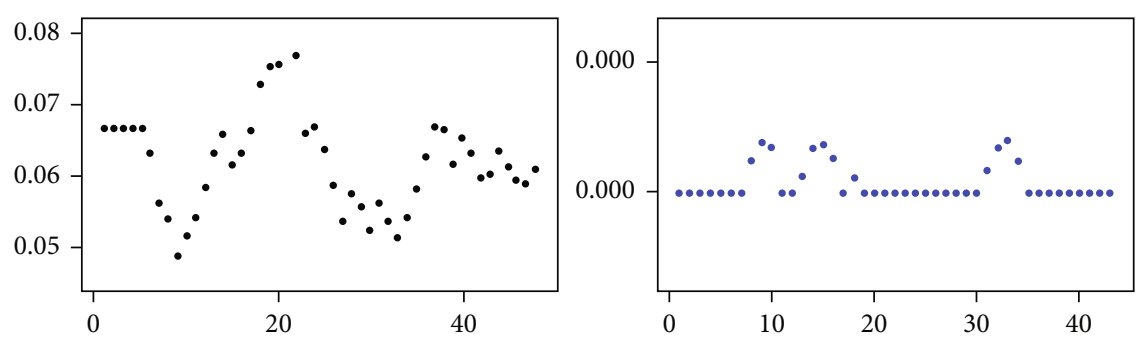

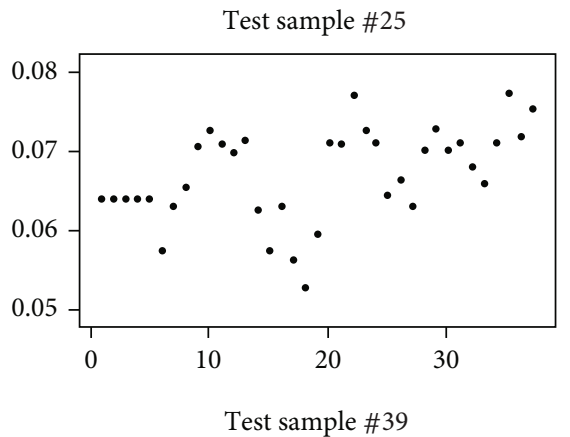

- SD value

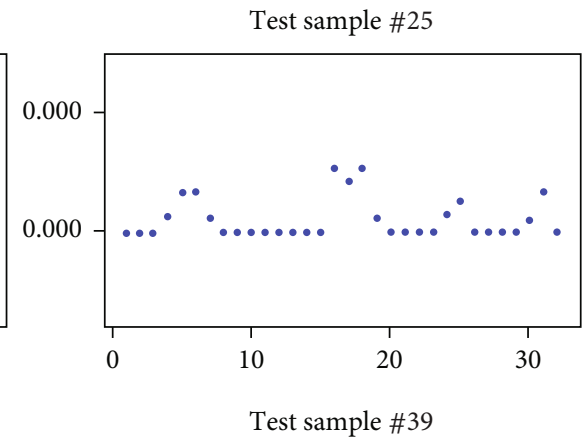

- ALWS value

FIGURE 9: SD and ALWS of test samples in the health stage.

TABle 6: Results of test examples in the health stage.

\begin{tabular}{lccccc}
\hline $\begin{array}{l}\text { Test sample } \\
\text { ID }\end{array}$ & $\begin{array}{c}\text { Current using } \\
\text { cycle }\end{array}$ & $\begin{array}{c}\text { Predict } \\
\text { RUL }\end{array}$ & $\begin{array}{c}\text { True } \\
\text { RUL }\end{array}$ & Error & Score \\
\hline$\# 25$ & 48 & 134 & 145 & -11 & 1.33 \\
$\# 39$ & 37 & 143 & 142 & 1 & 0.11 \\
\hline
\end{tabular}

assessment and prediction, accuracy and root mean square error (RMSE, as (12)) are used commonly. These symmetry indicators are feasible and easily accessible for evaluating the accuracy of RUL prediction results. However, for RUL prediction of aircraft engine, considering that the prediction result is greater than the actual available time will lead to maintenance decision fall behind the actual failure occurrence time, which will bring unacceptable unsafe consequences and economic loss. On the other hand, the prediction result is less than the actual value, and the result leads to advanced maintenance works and loss of useful life, which is safer and conservative. Therefore, in order to reasonable and acceptable RUL prediction results of the aircraft engine, an asymmetric indicator is used for evaluation in this paper, which is noted as the score function (calculated by (13)).

$$
\begin{gathered}
d=\mathrm{RUL}_{\text {predict }}-\mathrm{RUL}_{\text {true }}, \\
R M S E=\sqrt{\frac{1}{n} \sum d_{i}^{2}} \quad i=1,2, \cdots, n \\
s \begin{cases}e^{\left(-\frac{d}{13}\right)}-1, & \text { for } d<0 \\
e^{\left(\frac{d}{10}\right)}-1, & \text { for } d \geq 0\end{cases}
\end{gathered}
$$

\section{Implementation and Analysis}

4.1. Data Description. In this paper, the NASA's CMAPSS simulation data is selected to verify the proposed method. There are four types of training data and corresponding ground time from the beginning of the simulation to the occurrence of an engine failure. FD001 is the basic simulation data set with a single operating environment and one failure mode. FD002 has six operating environments and one failure mode. FD003 has a single operating environment and two failure modes. FD004 has six operating environments and two failure modes. The training data of CMAPSS is shown in Table 2.

In this study, the purpose is to establish degradation assessment and remaining life prediction model for the aircraft engine and is not focused on fault diagnosis and identification. Therefore, FD001 with a single operating environment and one failure mode is selected as the research data.

For any data sample, the parameter types and descriptions are the same, as shown in Table 3.

4.2. Feature Selection and Target Calculation. Feature selection is the essential and fundamental work for the proposed method. The Pseudocodes 1 shows there 21 sensors parameters in datasets, and one thing is important, these parameters are different in meaning, unit and value range.

For the training data FD001, to select features, as described in sectionII, the first task is to calculate the trend of parameters. The results of Mann Kendall test are shown in Table 4.

Pseudocode 2 shows that parameters ‘ 22 ," "s3," "s4," “s7," “s8," “s9" “s11," “s12," “s13," “s14," “s15," “s17," "s20," "s21"\} are selected as features, because these parameters have the monotonous trend, increasing, or decreasing, as bold objects in the table. 

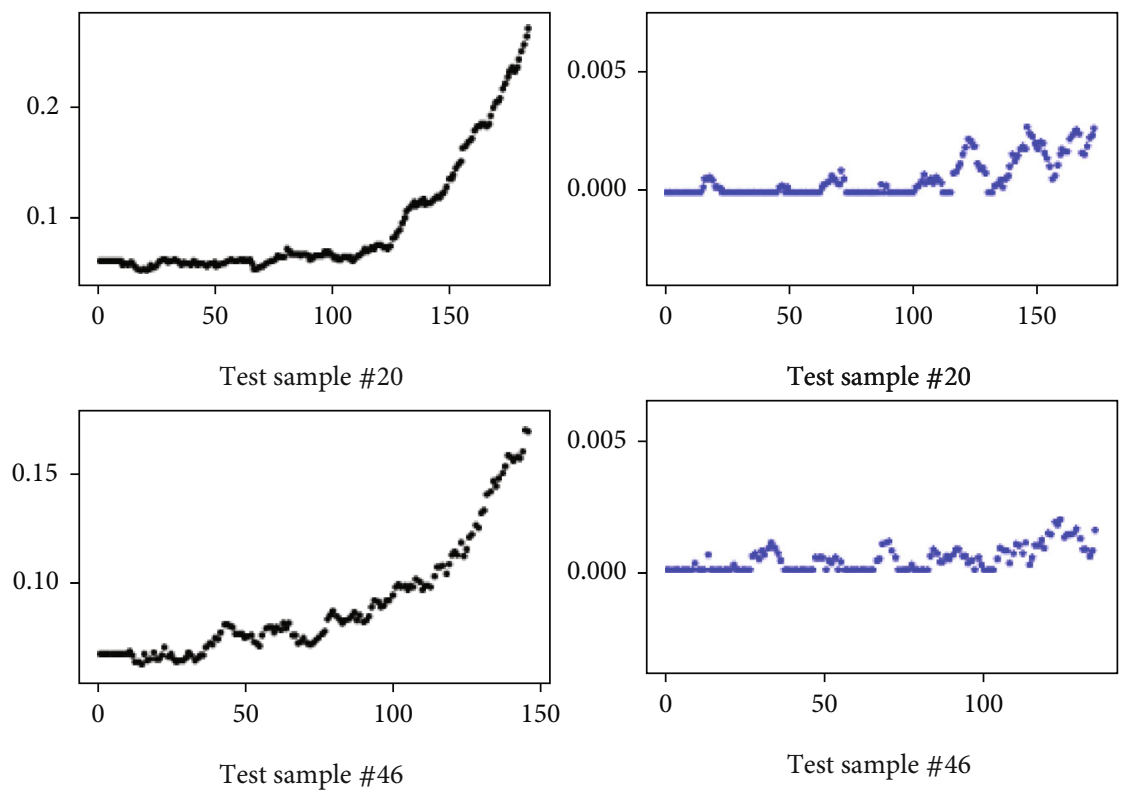

- SD value

- ALWS value

Figure 10: SD and ALWS of test samples in the degradation stage.

TABLE 7: Results of test examples in the degradation stage.

\begin{tabular}{lccccc}
\hline $\begin{array}{l}\text { Test sample } \\
\text { ID }\end{array}$ & $\begin{array}{c}\text { Current using } \\
\text { cycle }\end{array}$ & $\begin{array}{c}\text { Predict } \\
\text { RUL }\end{array}$ & $\begin{array}{c}\text { True } \\
\text { RUL }\end{array}$ & Error & Score \\
\hline$\# 20$ & 184 & 15 & 16 & -1 & 0.08 \\
$\# 46$ & 146 & 35 & 47 & -12 & 1.52 \\
\hline
\end{tabular}

The next important task is the calculation of the target value. Using D0 to represent the FD001 train data, all the first cycle of each engine in D0 is extracted and formed into the absolute healthy sample set $H$. Therefore, it is easy to get the healthy baseline of $H$.

For all samples in D0, based on the definition and calculation of SD, the SD value for each sample can be calculated. Let $L 1=w 1=10$, and SD value sequences of some engines in D0 are shown in Figure 5.

According to the definition and calculation of ALWS, the ALWS value of each engine can be calculated. Some samples of ALWS are shown in Figure 6.

For the life data in D0, the Kaplan Meier model is used to fit the survival process that is from the installation to the failure occurrence of an engine, as shown in Figure 7. The solid blue line in the figure is the survival curve, and the light blue band represents the 95\% confidence interval. With time increasing, the probability of survival $C(t)$ (in this study, the functioning engine is considered in survival) becomes smaller and smaller.

Based on the aforementioned SD-ALWS method, for historical datasets, it is feasible to divide D0 into D1 and D2 and represents the healthy datasets and the obvious degradation datasets, respectively, for the datasets D1 with healthy stage samples.

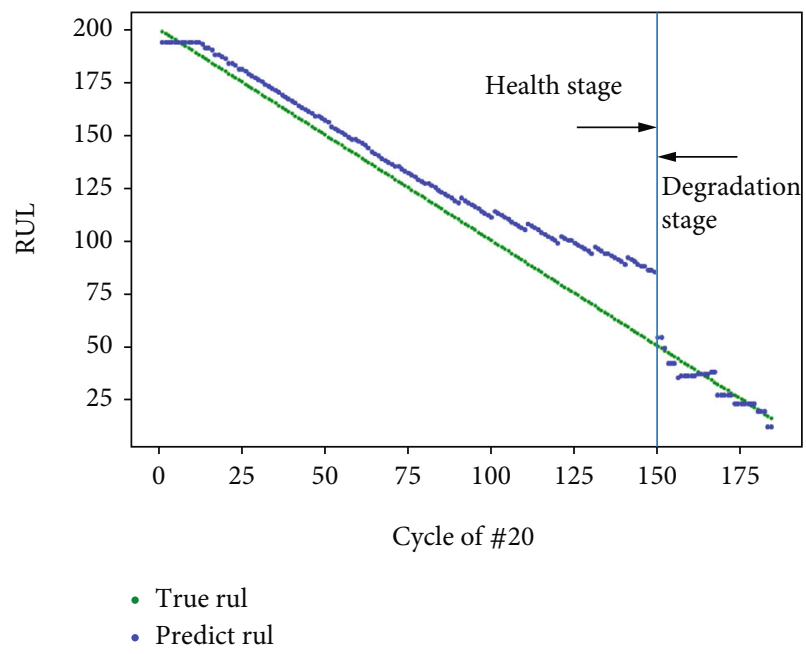

FIGURE 11: Complete RUL prediction process of test engine \#20.

For the datasets D2 with obvious degradation samples, $\mathrm{SD}$ is used as the independent variable, and $\mathrm{SOH}$ is used as the dependent variable to establish the mapping function between $\mathrm{SD}$ and $\mathrm{SOH}$. In this paper, several common and suitable functional forms are used to fit the mapping relationship, in which the hybrid function is composed of three basic functions, and the Levenberg-Marquardt method is selected to estimate parameters of each function. The fitting results of the mapping function are shown in the following Table 5 and Figure 8.

For data fitting, the $R$-square determination coefficient $\left(R^{2}\right)$ is a comprehensive evaluation index. As shown in the table, the hybrid function proposed in this paper performs best in these functions. Obviously, the value range of $\mathrm{SOH}$ is $(0,1)$. On the 


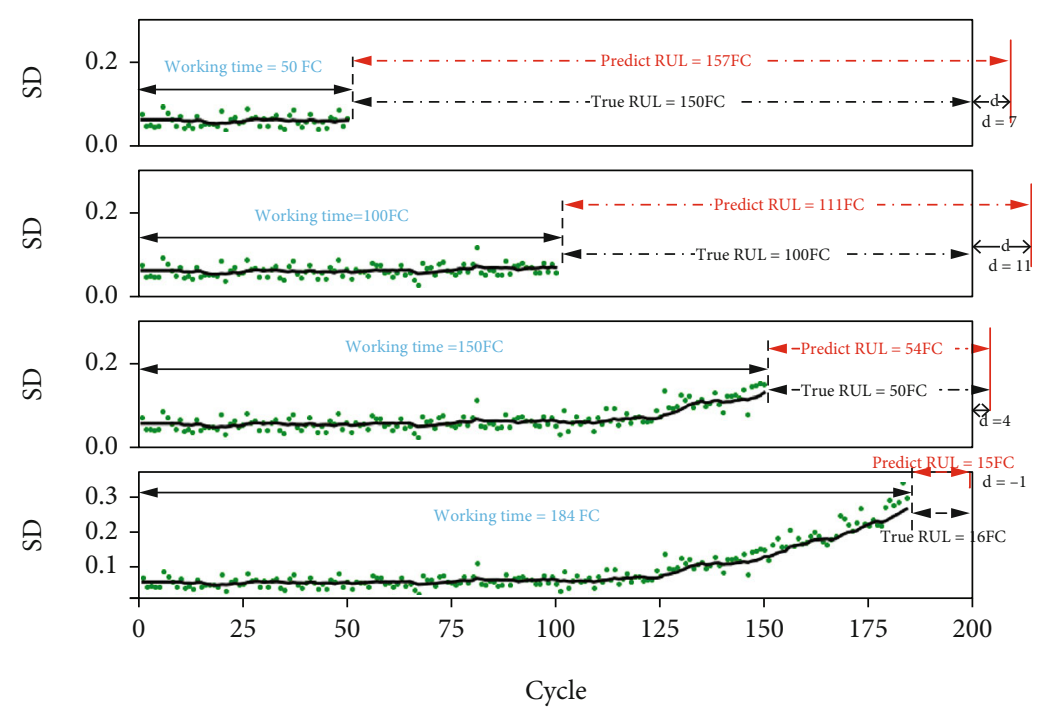

- SD value

FIGURE 12: SD and prediction RUL under different cycle of truncations of test engine \#20 in FD001.

TABLE 8: Results of all test samples.

\begin{tabular}{|c|c|c|c|c|c|}
\hline Test_id & Predict RUL & True RUL & $d=$ predict true & Score & $\mathrm{d} 2$ \\
\hline 1 & 96 & 91 & 5 & 0.65 & 25 \\
\hline 2 & 102 & 96 & 6 & 0.82 & 36 \\
\hline 3 & 115 & 124 & -9 & 1 & 81 \\
\hline 4 & 82 & 84 & -2 & 0.17 & 4 \\
\hline 5 & 87 & 87 & 0 & 0 & 0 \\
\hline 6 & 15 & 16 & -1 & 0.08 & 1 \\
\hline ... & $\ldots$ & $\ldots$ & $\ldots$ & ... & ... \\
\hline Total & - & - & - & 193.39 & $8886, \mathrm{RMSE}=13.33$ \\
\hline
\end{tabular}

other hand, from the view of the fitting diagram (Figure 8), there are some points that exceed the margin in the inverse function, power function, negative exponential function, and logarithmic function. Comprehensively consider the fitting effect and rationality, the proposed hybrid function is used to fit the mapping relationship between SD and SOH. For any moment $t$ of the test sample in gradation stage, the $\mathrm{SOH}$ can be calculated by the following formula (14).

$\mathrm{SOH}(t)=\operatorname{Exp}(-4.82 \times \mathrm{SD}(t))+\frac{0.015}{\mathrm{SD}(t)}+0.145 \times \operatorname{Ln}(\mathrm{SD}(t))$

4.3. Assessment and Prediction for New Observations. For any new test sample, $L$ and $w$ take the value 5 when the length of the sample is less than 100; otherwise, take the value to 10 . Therefore, referred to introductions in Section III, the SD and ALWS value can be calculated. Moreover, according the degradation assessment rule in Section III, the proposed SD-ALWS method can realize the performance evaluation of the test sample, that is, determining the stage of the sample.
4.3.1. For a Test Sample in the Health Stage. Taking engines \#25 and \#39 in test datasets as examples to illustrate calculation results of test samples in the health stage, the SD and ALWS are shown in Figure 9.

According to the rule, these two test samples are diagnosed as the healthy observation. With historical samples and life data reliability distribution $(C(t))$, according to formulae (5)(9), the RUL of test sample in the health stage can be predicted, and the results of the two samples are shown in Table 6.

From Table 6, the predict RUL of engine \#25 is less than true RUL; that is, the error is -11 ,which means the predicted life time before the actual life, in other words, that is a safe prediction. For \#39, the predict error is 1 , which means the predicted life time is late than the actual life and a potential accidental failure.

4.3.2. For a Test Sample in the Degradation Stage. Taking engine \#20 and \#46 in test datasets to illustrate calculation results of test samples in the degradation stage, the SD and ALWS are shown in Figure 10.

It can be seen from the figure that there is obvious upward trend of the SD, and the value of ALWS exceeds the threshold in the continuous detection window. 


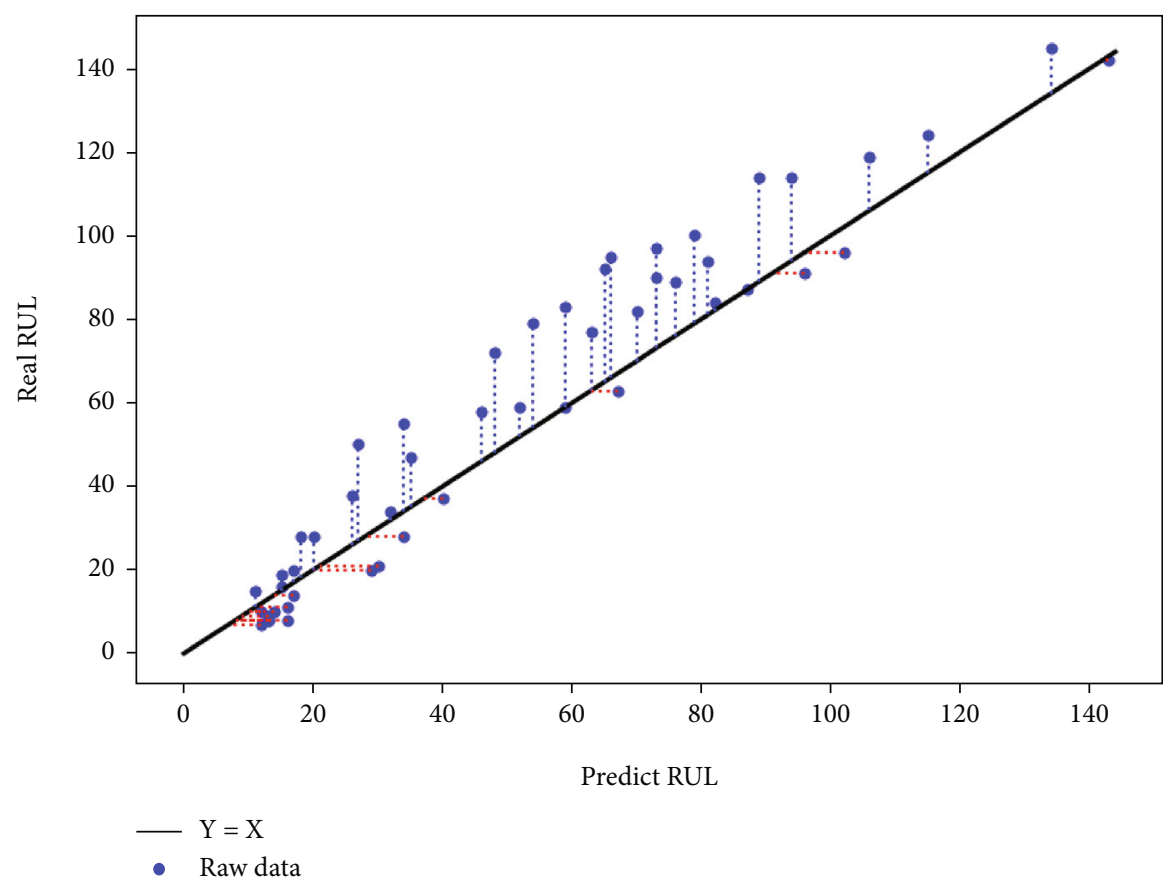

Figure 13: The results of RUL prediction of test samples in FD001.

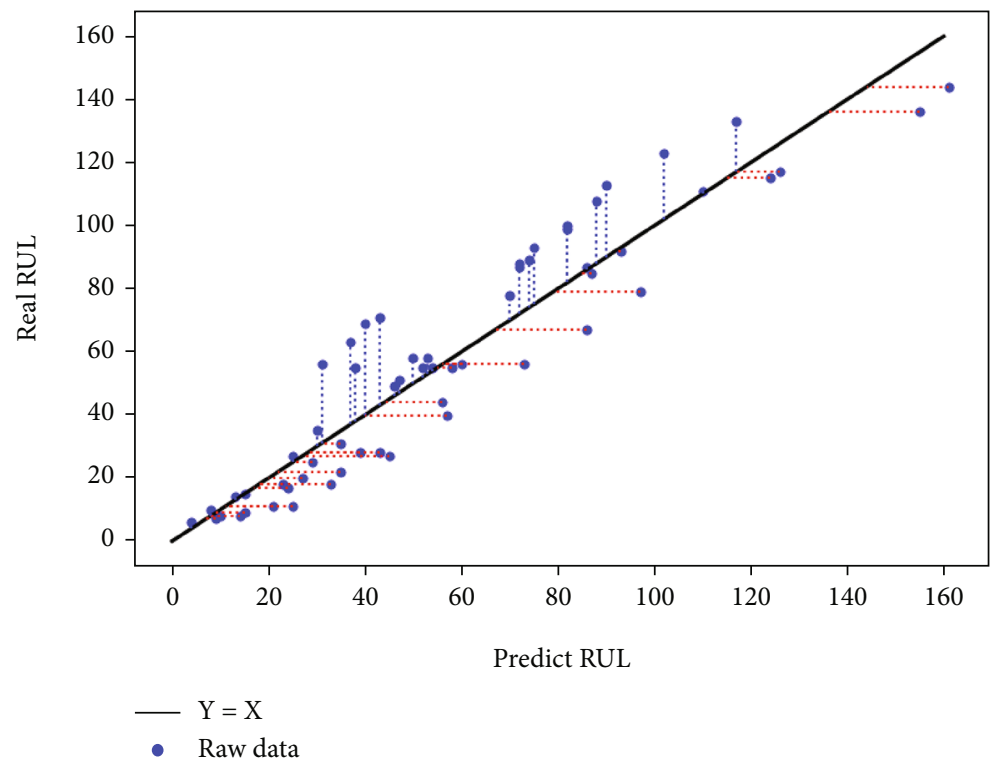

FIGURE 14: The results of RUL prediction of test samples in FD003.

According to the rule, these two test samples are diagnosed as the degraded observation. Furthermore, according to formulae (10) and (14), the RUL of test sample in the degradation stage can be predicted, and the results are shown in Table 7.

From Table 7 , the predict RUL of engine \#20 (as is shown in Figure 11) in the degradation stage is less than true RUL, which means the predicted life time before the actual life, in other words, that is a safe prediction.

As shown in Figure 11, the ordinate is the RUL, and the abscissa is using the cycle. On the figure of the RUL-using cycle, the prediction methods of two stages are different. Obviously, the prediction method based on similarity in the health stage causes greater deviation, which is related to the prior knowledge of historical samples. With the degradation of engine, it is critical to predict the accurate RUL for safety, and the new prediction method based on the mapping function can provide better results.

Furthermore, to illustrate the prediction effect of the proposed method on a single engine, Figure 12 shows SD and prediction RUL under different cycle of truncations ( $50 \mathrm{fc}, 100 \mathrm{fc}, 150 \mathrm{fc}$, the last flight cycle). It can be seen from 
TABLE 9: Comparison with other methods of predicting RUL for CMAPSS data.

\begin{tabular}{lcccc}
\hline \multirow{2}{*}{ Methods } & \multicolumn{2}{c}{ FD001 } & \multicolumn{2}{c}{ FD003 } \\
& Total score & RMSE & Total score & RMSE \\
\hline ARIMA-SVM [16] & N/A & 39.68 & N/A & N/A \\
BD-LSTM [24] & 338 & 16.14 & N/A & N/A \\
LSTM+GMM [34] & 308 & 14.08 & 221 & 12.15 \\
Light GBM [40] & 250 & 13.45 & N/A & N/A \\
Proposed method & 193.39 & 13.33 & 209.1 & 13.38 \\
\hline
\end{tabular}

the figure that the proposed method has a better prediction effect under the use cycle with a shorter real life.

4.4. Result Analysis and Discussion. For test samples in FD001, one thing is important that the degradation assessment is a meaningful work, but RUL prediction is our concern and the purpose of degradation assessment. The results of RUL prediction for test samples are shown in Table 8 and Figure 13.

From Figure 13, this black solid line $(Y=X)$ indicates that the predicted value is equal to the true value, and any point on this line indicates that the predicted result is completely consistent with the true value. The point below this line indicates that the predicted result is greater than the true value, which means the predicted failure time is too late. The point above this line indicates that the predicted result is less than the true value, which means the predicted failure time is too early. According to equations (12) and (13), the total score and RMSE of test samples in FD001 can be calculated, 193.39 and 13.33, respectively.

Because FD002 and FD004 have six operation environment types, it is difficult to extract the degradation feature using the proposed method. In order to further verify the model method on FD003, the RUL prediction results of test samples is shown in Figure 14.

Comparing Figures 13 and 14, for FD003 with two failure modes are mixed, the prediction results are also concentrated on the line $(Y=X)$, which means the proposed prediction method is effective for multiple failure mode samples. According to equations (12) and (13), the total score and RMSE of test samples in FD003 are 209.1 and 13.38, respectively.

In order to better highlight the proposed method, the RUL prediction results of this study are compared with other methods in related references, as shown in Table 9. It can be seen that the proposed method performs better on comprehensive indicators.

From Table 9, the proposed method has a lower total score in predicting RUL of single fault mode and multiple fault modes, which mean the prediction results are better than other methods in the table in score.

\section{Conclusions}

A novel method based on data fusion for aeroengine degradation assessment and RUL prediction is proposed in this paper, and the engineering feasibility is verified in the public simulation data. The key issue is to establish a two-stage performance degradation assessment and prediction model, which is by calculating the proposed health index and under the specific evaluation rule. Once a new observation is obtained, the new health status can be estimated by the proposed method, and furthermore, the corresponding residual life is calculated for different degradation stages.

For the prospect of practical application, the proposed two-stage degradation assessment model is more effective in detecting early failures, which is of great significance to the realization of condition-based maintenance and can avoid unplanned shutdowns and maintenance events caused by unexpected failures. For more accurate prediction results and better sensitivity, future research will focus on how to solve and quantify uncertain factors in the prediction process to enhance robustness.

\section{Data Availability}

Some or all data, models, or code generated or used during the study are available in a repository or online in accordance with funder data retention policies (Y. Liu, D. K. Frederick, J. A. Decastro, J. S. Litt, and W. W. Chan, "User's Guide for the Commercial Modular Aero-Propulsion System Simulation (C-MAPSS)," Nasa/Tm, vol. 2012-21743, no. March, pp. 1-40, 201) [29].

\section{Conflicts of Interest}

The authors declare that they have no conflicts of interest.

\section{Acknowledgments}

This work is supported by the Major Program of Civil Aviation Joint Funds of China (U1933202) and the Funding of Cultivation Project of Aero-Engine High Temperature Materials/Advanced Manufacturing \& Fault Diagnosis Science Foundation (91860139).

\section{References}

[1] M. Naeem, R. Singh, and D. Probert, "Consequences of aeroengine deteriorations for military aircraft," Applied Energy, vol. 70, no. 2, pp. 103-133, 2001.

[2] Y. G. Li and P. Nilkitsaranont, "Gas turbine performance prognostic for condition-based maintenance," Applied Energy, vol. 86, no. 10, pp. 2152-2161, 2009.

[3] M. Tahan, E. Tsoutsanis, M. Muhammad, and Z. A. Abdul Karim, "Performance-based health monitoring, diagnostics and prognostics for condition-based maintenance of gas turbines: a review," Applied Energy, vol. 198, pp. 122-144, 2017.

[4] H.-b. Peng, M.-m. Liu, and Y.-g. Wang, "Life prediction of engine based on take-off EGTM," Science and Technology, vol. 16, pp. 160-164, 2014.

[5] D. Chen and J. Sun, "Fuel and emission reduction assessment for civil aircraft engine fleet on-wing washing," Transportation Research Part D: Transport and Environment, vol. 65, pp. 324331, 2018.

[6] G. Ping, J. Chen, T. Pan, and J. Pan, "Degradation feature extraction using multi-source monitoring data via logarithmic 
normal distribution based variational auto-encoder," Computers in Industry, vol. 109, pp. 72-82, 2019.

[7] Z. Chen, S. Cao, and Z. Mao, "Remaining useful life estimation of aircraft engines using a modified similarity and supporting vector machine (SVM) approach," Energies, vol. 11, no. 1, p. 28, 2018.

[8] W. Peng, Y. F. Li, Y. J. Yang, J. Mi, and H. Z. Huang, "Bayesian degradation analysis with inverse Gaussian process models under time-varying degradation rates," IEEE Transactions on Reliability, vol. 66, no. 1, pp. 84-96, 2017.

[9] Y. Zhang, Wiener and Gamma Processes Overview for Degradation Modelling and Prognostic, M.S. thesis, NTNU, 2015.

[10] H. Wang, T. Xu, and Q. Mi, "Lifetime prediction based on Gamma processes from accelerated degradation data," Chinese Journal of Aeronautics, vol. 28, no. 1, pp. 172-179, 2015.

[11] W. Peng, Y. F. Li, Y. J. Yang, H. Z. Huang, and M. J. Zuo, "Inverse Gaussian process models for degradation analysis: a Bayesian perspective," Reliability Engineering \& System Safety, vol. 130, pp. 175-189, 2014.

[12] W. Wang, "A two-stage prognosis model in condition based maintenance," European Journal of Operational Research, vol. 182, no. 3, pp. 1177-1187, 2007.

[13] K. Liu, X. Hu, H. Zhou, L. Tong, D. Widanalage, and J. Marco, "Feature analyses and modelling of lithium-ion batteries manufacturing based on random forest classification," IEEE/ASME Transactions on Mechatronics, vol. 99, 2021.

[14] D. Feng, M. Xiao, Y. Liu, H. Song, Z. Yang, and L. Zhang, “A kernel principal component analysis-based degradation model and remaining useful life estimation for the turbofan engine," Advances in Mechanical Engineering, vol. 8, no. 5, 2016.

[15] P. J. García Nieto, E. García-Gonzalo, F. Sánchez Lasheras, and F. J. de Cos Juez, "Hybrid PSO-SVM-based method for forecasting of the remaining useful life for aircraft engines and evaluation of its reliability," Reliability Engineering \& System Safety, vol. 138, pp. 219-231, 2015.

[16] C. Ordóñez, F. Sánchez Lasheras, J. Roca-Pardiñas, and F. J. . C. Juez, "A hybrid ARIMA-SVM model for the study of the remaining useful life of aircraft engines," Journal of Computational and Applied Mathematics, vol. 346, pp. 184-191, 2019.

[17] F. Xue, P. Bonissone, A. Varma, W. Yan, N. Eklund, and K. Goebel, "An instance-based method for remaining useful life estimation for aircraft engines," Journal of Failure Analysis and Prevention, vol. 8, no. 2, pp. 199-206, 2008.

[18] F. Lu, J. Wu, J. Huang, and X. Qiu, “Aircraft engine degradation prognostics based on logistic regression and novel OSELM algorithm," Aerospace Science and Technology, vol. 84, pp. 661-671, 2019.

[19] R. Khelif, B. Chebel-Morello, S. Malinowski, E. Laajili, F. Fnaiech, and N. Zerhouni, "Direct remaining useful life estimation based on support vector Regression," IEEE Transactions on Industrial Electronics, vol. 64, no. 3, pp. 2276-2285, 2017.

[20] K. Liu, Y. Shang, Q. Ouyang, and W. D. Widanage, "A datadriven approach with uncertainty quantification for predicting future capacities and remaining useful life of lithium-ion battery," IEEE Transactions on Industrial Electronics, vol. 99, 2021.

[21] S. Khan and T. Yairi, "A review on the application of deep learning in system health management," Mechanical Systems and Signal Processing, vol. 107, pp. 241-265, 2018.
[22] A. Listou Ellefsen, E. Bjørlykhaug, V. Æsøy, S. Ushakov, and H. Zhang, "Remaining useful life predictions for turbofan engine degradation using semi- supervised deep architecture," Reliability Engineering \& System Safety, vol. 183, pp. 240-251, 2019.

[23] X. Li, Q. Ding, and J. Q. Sun, "Remaining useful life estimation in prognostics using deep convolution neural networks," Reliability Engineering \& System Safety, vol. 172, pp. 1-11, 2018.

[24] S. Zheng, K. Ristovski, A. Farahat, and C. Gupta, "Long shortterm memory network for remaining useful life estimation," in 2017 IEEE international conference on prognostics and health management (ICPHM), pp. 88-95, Dallas, TX, USA, 2017.

[25] J. Zhang, P. Wang, R. Yan, and R. X. Gao, "Long short-term memory for machine remaining life prediction," Journal of Manufacturing Systems, vol. 48, pp. 78-86, 2018.

[26] H. Yan, J. Sun, and H. Zuo, "Anomaly detection based on multivariate data for the aircraft hydraulic system," Proceedings of the Institution of Mechanical Engineers Part I Journal of Systems and Control Engineering, vol. 235, no. 5, pp. 593-605, 2021.

[27] Q. Zheng, H. Zhang, Y. Li, and Z. Hu, “Aero-engine on-board dynamic adaptive MGD neural network model within a large flight Envelope," IEEE Access, vol. 6, pp. 45755-45761, 2018.

[28] K. Liu, Z. Wei, Z. Yang, and K. Li, "Mass load prediction for lithium-ion battery electrode clean production: A machine learning approach," Journal of Cleaner Production, vol. 289, no. 1, p. 125159, 2021.

[29] Y. Liu, D. K. Frederick, J. A. Decastro, J. S. Litt, and W. W. Chan, "User's guide for the commercial modular aeropropulsion system simulation (C-MAPSS)," Nasa/Tm, vol. 2012, article 21743, 2012.

[30] A. Mosallam, K. Medjaher, and N. Zerhouni, "Data-driven prognostic method based on Bayesian approaches for direct remaining useful life prediction," Journal of Intelligent Manufacturing, vol. 27, no. 5, pp. 1037-1048, 2016.

[31] L. Liu, S. Wang, D. Liu, Y. Zhang, and Y. Peng, "Entropy-based sensor selection for condition monitoring and prognostics of aircraft engine," Microelectronics Reliability, vol. 55, no. 9-10, pp. 2092-2096, 2015.

[32] P. Wen, S. Zhao, S. Chen, and Y. Li, "A generalized remaining useful life prediction method for complex systems based on composite health indicator," Reliability Engineering \& System Safety, vol. 205, article 107241, 2021.

[33] S. Behera, R. Misra, and A. Sillitti, "Multiscale deep bidirectional gated recurrent neural networks based prognostic method for complex non-linear degradation systems," Information Sciences, vol. 554, pp. 120-144, 2021.

[34] M. Sayah, D. Guebli, Z. Noureddine, and Z. al Masry, "Deep LSTM enhancement for RUL prediction using Gaussian mixture models," Automatic Control and Computer Sciences, vol. 55, no. 1, pp. 15-25, 2021.

[35] A. Elsheikh, S. Yacout, and M. Ouali, "Bidirectional handshaking LSTM for remaining useful life prediction," Neurocomputing, vol. 323, pp. 148-156, 2019.

[36] S. Pillai and P. Vadakkepat, "Two stage deep learning for prognostics using multi-loss encoder and convolutional composite features," Expert Systems with Applications, vol. 171, p. 114569, 2021.

[37] Z. Liang, J. Gao, H. Jiang, X. Gao, Z. Gao, and R. Wang, “A degradation degree considered method for remaining useful life prediction based on similarity," Computing in Science \& Engineering, vol. 21, no. 1, pp. 50-64, 2019. 
[38] X. Jia, M. Zhao, Y. Di, Q. Yang, and J. Lee, “Assessment of data suitability for machine prognosis using maximum mean discrepancy," IEEE Transactions on Industrial Electronics, vol. 65, no. 7, pp. 5872-5881, 2018.

[39] A. Gretton, K. M. Borgwardt, and M. J. Rasch, "A kernel twosample test," The Journal of Machine Learning Research, vol. 13, no. 1, pp. 723-773, 2012.

[40] F. Li, "A light gradient boosting machine for remainning useful life estimation of aircraft engines," in IEEE Conf. Intell. Transp. Syst. Proceedings, ITSC, 2018, pp. 3562-3567, Novem, 2018. 